\title{
Function and transcriptional regulation of TCTN1 in oral squamous cell carcinoma
}

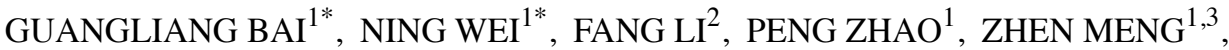 \\ BO ZOU ${ }^{1}$, YUJIAO LIU ${ }^{1}$, KAI XU ${ }^{1}, \mathrm{KEYI} \mathrm{LI}^{1}, \mathrm{CHAO} \mathrm{YAO}^{4}$ and PENG YANG ${ }^{1}$ \\ ${ }^{1}$ Department of Stomatology and Key Laboratory of Precision Biomedicine (Liaocheng), \\ Liaocheng People's Hospital, Medical College of Liaocheng University, Liaocheng, Shandong 252000; \\ ${ }^{2}$ Clinical Laboratory, Heze Municipal Hospital, Heze, Shandong 274000; ${ }^{3}$ Translational Medicine Research Center, \\ Medical College of Liaocheng University, Liaocheng, Shandong 252000; ${ }^{4}$ Department of Oral and Maxillofacial Surgery, \\ Jinan Stomatological Hospital, Jinan, Shandong 250000, P.R. China
}

Received August 17, 2021; Accepted November 10, 2021

DOI: 10.3892/or.2021.8237

\begin{abstract}
Oral squamous cell carcinoma (OSCC) is one of the most common types of head and neck squamous cell carcinoma (HNSCC) with a poor survival rate. In the present study, the involvement of tectonic 1 (TCTN1) in OSCC was explored. The relevance between TCTN1 and HNSCC clinicopathological features was first analyzed and it was revealed that TCTN1 was associated with the tumor clinical stage and grade. In in vitro experiments, it was demonstrated that the proliferative, migratory and invasive capacity of OSCC CAL27 cells and SCC15 cells was significantly suppressed due to TCTN1 knockdown. Additionally, the core promoter of TCTN1 was confirmed and transcription factor AP-2 alpha (TFAP2A) was suggested as a regulator of TCTN1 mRNA expression. On the whole, the present study elucidated the direct association between TCTN1 and OSCC for the first time, to the best of our knowledge, and the TFAP2A/TCTN1 axis was suggested as a potential novel therapeutic target for OSCC.
\end{abstract}

Correspondence to: Dr Chao Yao, Department of Oral and Maxillofacial Surgery, Jinan Stomatological Hospital, 101 Jingliu Road, Jinan, Shandong 250000, P.R. China

E-mail: yaochaojinankq@126.com

Dr Peng Yang, Department of Stomatology and Key Laboratory of Precision Biomedicine (Liaocheng), Liaocheng People's Hospital, Medical College of Liaocheng University, 67 Dongchangxi Road, Liaocheng, Shandong 252000, P.R. China

E-mail: yangpenglcrmyy@163.com

${ }^{*}$ Contributed equally

Key words: oral squamous cell carcinoma, tectonic 1, transcription factor AP-2 alpha, promoter, transcription

\section{Introduction}

Oral squamous cell carcinoma (OSCC), characterized by an increased morbidity and mortality, is one of the main types of head and neck squamous cell carcinoma (HNSCC) (1). Several mechanisms involved in the progression of OSCC have been revealed; however, these mechanisms are not yet fully understood. Thus, the deeper understanding of the regulatory mechanisms of OSCC is of utmost importance.

Tectonic family member 1 (TCTN1), belonging to the TCTN family, is a signal-sequence-containing secreted and transmembrane protein (2). TCTN1 participates in several physiological processes and pathological processes, including ciliogenesis, Varadi syndrome, Joubert syndrome and non-motile ciliopathies (3-6). TCTN1 is involved in the development and progression of various tumors. The silencing of TCTN1 has been reported to inhibit proliferation, and induce cell cycle arrest and apoptosis in human thyroid cancer (7). Furthermore, it has been revealed that TCTN1 knockdown may significantly inhibit colon cancer cell growth (8). Furthermore, TCTN1 is associated with prostate cancer cell growth and migration (9). Therefore, TCTN1 has been suggested to be a potential target for cancer therapy. TCTN1 participates in the cell growth and apoptosis of esophageal squamous cell carcinoma, a cell type of HNSCC (10). However, although OSCC is a main type of HNSCC, little is known about TCTN1 involvement in OSCC progression. Additionally, the mechanisms through which TCTN1 expression is regulated remain largely unknown.

The UALCAN database is a comprehensive web resource for analyzing cancer OMICS data, which can be used to perform pan-cancer gene expression analysis and to analyze clinicopathological feature information (11). In the present study, TCTN1 expression in HNSCC was analyzed and evaluated in each HNSCC tumor stage and grade. OSCC is a main type of HNSCC, and therefore, it may be possible that the TCTN1 expression levels in OSCC may be similar to those of HNSCC. Thus, TCTN1 expression analysis was evaluated in HNSCC and OSCC. 
Transcription is the process through which RNA is synthesized according to genomic DNA; thus, the genetic information is transduced from DNA to RNA (12). Transcriptional regulation is an important part of gene expression regulation. Transcription is very complex and often requires the assistance of many factors, including long non-coding RNAs (lncRNAs), microRNAs (miRNAs) and transcription factors (13-15). Transcription factors are a group of proteins which can bind to specific sequences upstream of target genes inducing gene upregulation or downregulation (16-18). Currently, there is limited information available on TCTN1 transcriptional regulation. Several software applications may be used for the prediction of putative transcription factors binding to target genes, according to the promoter sequence, including PROMO and JASPAR2020. PROMO is a virtual laboratory for identifying putative transcription factor binding sites based on DNA sequences (http://alggen.lsi.upc.es/cgi-bin/promo_ v3/promo/promoinit.cgi?dirDB=TF_8.3) (19). JASPAR2020 software provides a high-quality transcription factor binding profile database (http://jaspar.genereg.net/) (20).

In the present study, the association of TCTN1 with HNSCC tumor stage and grade was first analyzed using bioinformatics analysis. Subsequently, the regulatory effects of TCTN1 on OSCC cell proliferation, migration and invasion were evaluated and it was revealed that an important transcription factor, transcription factor AP-2 alpha (TFAP2A), may promote TCTN1 expression.

\section{Materials and methods}

Cell culture and clinical specimens. The CAL27 (cat. no. 1101HUM-PUMC000338), SCC15, and SCC9 cells were purchased from the National Biomedical Laboratory Cell Bank of China. The SCC15 (cat. no. bio-69136) and SCC9 cells (cat. no. bio-69190) were purchased from Biobw (https://www.biobw.org/). Tca83 cells were kindly provided by Professor Ye-Hua Gan of the Stomatological Hospital of Peking University (21). Normal human oral keratinocytes (HOK cells) were purchased from ScienCell Research Laboratories, Inc. (cat. no. 2610). All cell lines were cultured in Dulbecco's modified Eagle's medium (DMEM) (Gibco; Thermo Fisher Scientific, Inc.), containing $10 \%$ fetal bovine serum (FBS) (Gibco; Thermo Fisher Scientific, Inc.) in an incubator (Thermo Fisher Scientific, Inc.) with $5 \% \mathrm{CO}_{2}$ at $37^{\circ} \mathrm{C}$. Trypsin-EDTA reagent (Gibco; Thermo Fisher Scientific, Inc.) was used for cell digestion. Following digestion, cells were seeded in a 96- or 12-well plate for cell proliferation, migration and invasion assay. All seeding numbers for each assay are described below.

OSCC and adjacent normal tissues were obtained from 21 patients at Liaocheng People's Hospital (Liaocheng). All patient information is presented in Table SI. The tissue collection was checked and approved by the Ethics Committee of Liaocheng People's Hospital (Approval No. LC202176). In total, 21 paired OSCC and adjacent normal tissues were used in the present study. All patients provided written informed consent.

Analysis of the association between TCTN1 and clinicopathological features. The association between TCTN1 and the patient clinicopathological features was evaluated using the UALCAN database (http://ualcan.path.uab.edu/). The UALCAN database is a comprehensive web resource for analyzing cancer OMICS data, which can be used for performing pan-cancer gene expression analysis and analyzing clinicopathological feature information (11).

Reverse transcription-quantitative PCR (RT-qPCR). CAL27, SCC15, Tca83 and SCC9 cell RNA, as well as clinical specimen RNA was extracted using TRIzol ${ }^{\circledR}$ reagent (Thermo Fisher Scientific, Inc.). After the concentration was measured using a ultrafine ultraviolet spectrophotometer (Thermo Fisher Scientific, Inc.) according to the absorbance at $260 \mathrm{~nm}, 2 \mu \mathrm{g}$ RNA was used for cDNA synthesis using the BeyoRT ${ }^{\mathrm{TM}}$ II M-MLV Reverse Transcriptase kit (cat. no. D7160L, Beyotime Institute of Biotechnology), according to the manufacturer's instructions. qPCR was performed using a BeyoFast ${ }^{\mathrm{TM}}$ SYBR-Green qPCR Mix kit (cat. no. D7260, Beyotime Institute of Biotechnology) according to the manufacturer's instructions. All RT-qPCR experiments were performed on an ABI 7500 Real-Time PCR instrument (Applied Biosystems; Thermo Fisher Scientific, Inc.) with the following conditions: $95^{\circ} \mathrm{C}, 10 \mathrm{~min} ; 95^{\circ} \mathrm{C}, 15 \mathrm{sec} ; 60^{\circ} \mathrm{C}, 60 \mathrm{sec}$ for 40 cycles. The primers used for RT-qPCR are listed in Table SII. $\beta$-actin was used as an internal control. The expression analysis method used was $2^{-\Delta \Delta \mathrm{Cq}}(22)$.

TCTN1 knockdown and TFAP2A overexpression. TCTN1 knockdown was performed by infecting the CAL27 cells and SCC15 cells with shTCTN1 lentiviral construct which targets the TCTN1 sequence (5'-GAGAAGGAACTGATGCAT CTGAGC-3'); the cells transfected with the shCon lentiviral construct, which has no target sequence in cells, was used as a negative control. The corresponding shRNA sequences were as follows: sh-TCTN1, 5'-GCTCAGATGCATCAGTTCCTT CTCGAGAAGGAACTGATGCATCTGAGCTTTTTT-3'; and shCon, 5'-AACAAGATGAAGAGCACCAACTCGAGTTGG TGCTCTTCATCTTGTTG-3'. TFAP2A overexpression was performed by infecting the CAL27 cells and SCC15 cells with the lv-oeTFAP2A lentiviral construct, and the cells transfected with the lv-oeCon lentiviral construct were used as negative control. lv-oeTFAP2A was constructed by inserting the TFAP2A cDNA into the lentivirus; lv-oeCon was constructed by inserting the fragment with the sequence as TFAP2A cDNA, except that all the ATG was replaced by AAG to abolish the transcription. The sequences of the overexpression vector and the control are presented as supplementary material (Data SI). Both constructs were designed and synthesized at Genechem, Inc., based on a 3rd generation lentiviral system. Briefly, $1.5 \mu \mathrm{g}$ vector and $1.5 \mu \mathrm{g}$ were transfected into 293T cells (cat. no. bio-12947, Biobw; https://www.biobw.org/) with Lipofectamine $3000^{\circledR}$ reagent (cat. no. L3000001; Thermo Fisher Scientific, Inc.) for $24 \mathrm{~h}$ at $37^{\circ} \mathrm{C}$, and the medium was then collected and the virus was harvested by ultracentrifugation at $80,000 \times \mathrm{g}, 4^{\circ} \mathrm{C}$ for $2 \mathrm{~h}$ in a Optima XPN centrifuge (Beckman, Coulter, Inc.). For lentivirus transduction, the CAL27 cells and SCC15 cells were seeded in a 12-well plate at 30,000 cells/well. Subsequently, at the logarithmic cell growth phase, the lentiviral constructs (shCon or shTCTN1) with a MOI of 10 were added into the well with the assistance of $2 \mu \mathrm{l}$ polybrane reagent (Cat No. H8761, Beijing Solarbio Science \& 
Technology Co., Ltd.). After $24 \mathrm{~h}$, the medium was replaced to wipe off the residual constructs. After the cells were cultured for a further $48 \mathrm{~h}$, cells transfected with lentiviral constructs were selected using puromycin (MilliporeSigma). Due to the green fluorescent protein (GFP) gene inserted in the lentiviral DNA, green fluorescence detected using an IX53 inverted fluorescence microscope (IX53, Olympus Corporation) was used to identify whether the cells were transfected with lentivirus or not. RT-qPCR was used to confirm whether TCTN1 was effectively knocked down.

Western blot analysis. The CAL27 cells and SCC15 cells were lysed using RIPA reagent (Applygene). The cells lysates were centrifuged at $12,000 \mathrm{xg}$ at $4^{\circ} \mathrm{C}$ for $4 \mathrm{~h}$. The supernatants were collected, and the concentration was measured using a BCA kit (Invitrogen; Thermo Fisher Scientific, Inc.). Subsequently, $50 \mu \mathrm{g}$ protein were subjected to $10 \%$ SDS-PAGE electrophoresis and then transferred onto a nitrocellulose (NC) membrane (MilliporeSigma). After the NC membrane was blocked in 5\% fat-free milk (Applygene) at $20^{\circ} \mathrm{C}$ for $1 \mathrm{~h}$, the membrane was incubated with the primary antibodies $(1: 1,000$ diluted into TBST) for $12 \mathrm{~h}$ at $4^{\circ} \mathrm{C}$. The membrane was then incubated with HRP-conjected goat anti-mouse secondary antibodies (A0216, Beyotime Institute of Biotechnology) or HRP-conjected goat anti-rabbit secondary antibodies (A0208, Beyotime Institute of Biotechnology) (1:10,000 diluted into TBST) for $1 \mathrm{~h}$ at $20^{\circ} \mathrm{C}$. Finally, the membrane was immersed in ECL luminous fluid (Beyotime Institute of Biotechnology) and detected using an automatic chemiluminescence image analysis system (Tanon4800, Tanon Science and Technology Co., Ltd.). z. The anti-MMP-9 (\#13667), anti-cyclin D1 antibodies (\#2922) and anti- $\beta$-actin antibodies (\#3700S) were purchased from Cell Signaling Technology, Inc. $\beta$-actin was used as internal control.

Cell Counting Kit-8 (CCK-8) assay. Cell proliferation capacity was evaluated using CCK-8 assay. The CAL27 and SCC15 cells were seeded in 96-well plates at 3,000 cells/well, and then cultured in the incubator with $5 \% \mathrm{CO}_{2}$ at $37^{\circ} \mathrm{C}$. At 0,24 and $48 \mathrm{~h}$ of culture, $10 \mu \mathrm{l}$ CCK- 8 reagent (Dojindo Molecular Technologies, Inc.) were added to the cells. Following a 2-h incubation at $37^{\circ} \mathrm{C}$, the absorbance at $\mathrm{OD}_{595 \mathrm{~nm}}$ was measured using a Multiskan spectrophotometer (Multiskan, Thermo Fisher Scientific, Inc.).

Transwell assay. Transwell assay was performed to evaluate the migratory and invasive capacity of the CAL27 cells and SCC15 cells. In order to evaluate migration, 100,000 cells were seeded into the upper chamber of a Transwell plate (cat. no. 3460, Corning, ME, USA) with DMEM containing no FBS, while the lower chamber was supplemented with DMEM containing $15 \%$ FBS. Following a 24 -h culture at $37^{\circ} \mathrm{C}$, the cells on the upper chamber were wiped away using a cotton swab, and cells passing through the membrane were stained with crystal violet (Beyotime Institute of Biotechnology) at $25^{\circ} \mathrm{C}$ for $3 \mathrm{~min}$. The cells were photographed, and the cell number was counted under a T2R inverted microscope (T2R, Nikon Corporation) at x200 magnification and counted in five randomly selected visual fields. The protocol for the evaluation of cell invasion was similar to the migration evaluation protocol, except from the following differences: The upper chambers were pre-coated with $20 \mu \mathrm{g}$ extracellular matrix gel (MilliporaSigma) at $37^{\circ} \mathrm{C}$ for $2 \mathrm{~h}$ and the cells were subsequently seeded at a concentration of 200,000 cells/well.

Construction of TCTN1 promoter-reporter. The human TCTN1 promoter sequence was obtained from GenBank (https://www.ncbi.nlm.nih.gov/gene/79600). The putative full-length promoter $(-1,500$ to $+50 \mathrm{bp}$, transcription starting site was defined as +1 ) of human TCTN1 was amplified from the genomic DNA of CAL27 cells using regular PCR with the following conditions: $95^{\circ} \mathrm{C}, 1 \mathrm{~min} ; 95^{\circ} \mathrm{C} 15 \mathrm{sec} ; 55^{\circ} \mathrm{C}, 30 \mathrm{sec}$; $72^{\circ} \mathrm{C}, 1 \mathrm{~min}$ for 30 cycles. The sequences of the primers used are listed in Table SIII. Following an agarose gel electrophoresis, the promoter segment was extracted using an Agarose Gel Extraction kit (DH101-01, Biomed; http://www.biomed168. $\mathrm{com} /$ ) and was cut by two restriction enzymes, SacI and MluI. Subsequently, the promoter segment was inserted pGL3-basic vector (Addgene, Inc.) which was also cut by $S a c \mathrm{I}$ and $M l u \mathrm{I}$. The promoter reporter was confirmed by Sanger sequencing on an Illumina NextSeq 500 instrument (Illumina) at Sangon Biotech Co., Ltd. and was named pGL3-TCTN1. The primer used for DNA sequencing was: 5'-CTAGCAAAATAGGCT GTCCC-3'. All the sequences of the TCTN1 promoter regaions are provided as supplementary material in Data SI.

Construction of deletion mutants for the TCTN1 promoter. The pGL3-TCTN1 plasmid was used as the template. The primer sequences were designed alongside the deleted region and are listed in the Table SIII. The PCR cycling was performed on an ABI Veriti PCR thermocycle instrument (Applied Biosystems) with the conditions as follows: $95^{\circ} \mathrm{C}, 1 \mathrm{~min} ; 95^{\circ} \mathrm{C}, 15 \mathrm{sec} ; 55^{\circ} \mathrm{C}$, $30 \mathrm{sec} ; 72^{\circ} \mathrm{C}, 5 \mathrm{~min}$ for 30 cycles; $72^{\circ} \mathrm{C}, 10 \mathrm{~min}$. Subsequently, the product was extracted using an Agarose Gel Extraction kit (Biomed, http://www.biomed168.com/). Following incubation with T4 polynucleotide kinase (New England Biolabs, Inc.) at $37^{\circ} \mathrm{C}$ for $1 \mathrm{~h}$, the product was self-linked using a T4 DNA ligase (New England Biolabs, Inc.) at $37^{\circ} \mathrm{C}$ for $1 \mathrm{~h}$ and transferred into the top 10 competent bacterial $E$. coli cells (Biomed) for amplification. TCTN1 promoter deletion mutants were confirmed by Sanger sequencing in an Illumina NextSeq 500 instrument (Illumina, CA, USA) at Sangon Biotech Co., Ltd. The primer used for DNA sequencing was: 5'-CTAGCAAAATAGGCTGTCCC-3'.

Site-directed mutagenesis. Site-directed mutagenesis was performed according to a previous study (23). Firstly, PCR with pGL3-TCTN1 (-550/-491) as the template was performed, by using a Q5 high-fidelity DNA polymerase (\#E0555L, New England Biolabs, Inc.) in an ABI Veriti PCR thermocycle instrument (Applied Biosystems) according to the following protocol: $95^{\circ} \mathrm{C}, 1 \mathrm{~min} ; 95^{\circ} \mathrm{C}, 15 \mathrm{sec} ; 50^{\circ} \mathrm{C}, 30 \mathrm{sec} ; 72^{\circ} \mathrm{C}, 5 \mathrm{~min}$ for 30 cycles; $72^{\circ} \mathrm{C}, 10 \mathrm{~min}$. The primers used for mutating the TFAP2A-binding site are as follows: Forward, 5'-ACTGCACTC CAATTGTTTATACAGAGTGAG-3' and reverse, 5'-CTCACT CTGTATAAACAATTGGAGTGCAGT-3'. The products were digested with DpnI enzyme (New England Biolabs, Inc.) and then transferred into the top 10 bacterial E. coli cells (Biomed) for amplification. Mutations were confirmed by Sanger sequencing.

Plasmid transfection. The TCTN1 promoter plasmid was transfected into the CAL27 cells or SCC15 cells using 
A

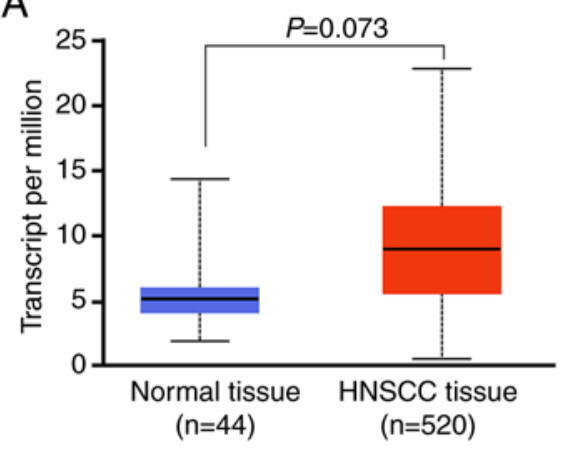

B

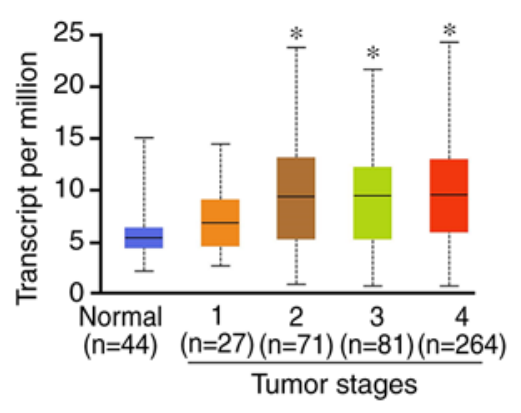

C

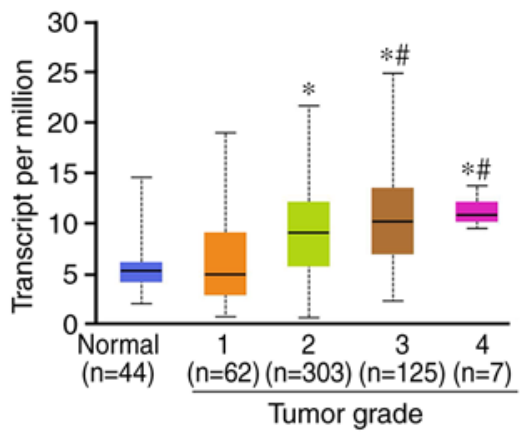

D

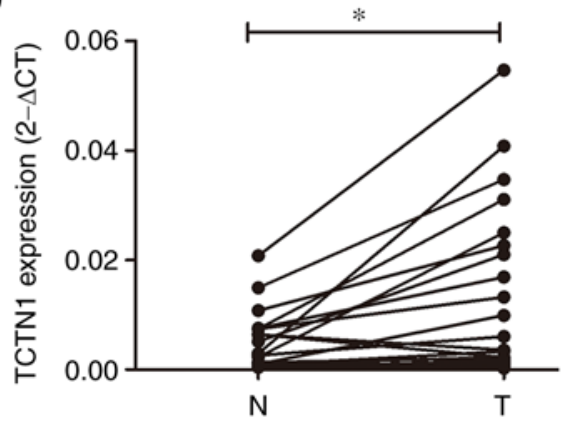

E

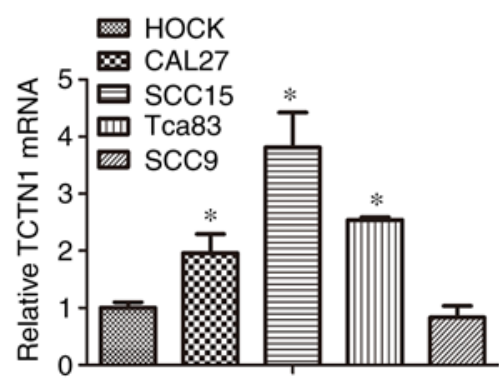

Figure 1. TCTN1 expression in HNSCC tissues. (A) TCTN1 expression in normal tissues and HNSCC tissues based on the UALCAN database. (B) TCTN1 expression in different tumor stages of HNSCC. ${ }^{*} \mathrm{P}<0.05$ vs. normal. (C) TCTN1 expression in different tumor grades of HNSCC. $\mathrm{P}<0.05$ vs. normal, ${ }^{\#} \mathrm{P}<0.05$ vs. grade 1. (D) Expression of TCTN1 in 21 OSCC tissues and paired tumor-adjacent normal tissues was detected by using RT-qPCR. "P<0.05. N, adjacent normal tissue; T, tumor tissue of OSCC. (E) Expression of TCTN1 in HOK, CAL27, SCC15, Tca83 and SCC9 cells was detected using RT-qPCR. ${ }^{*} \mathrm{P}<0.05$. TCTN1, tectonic family member 1 ; HNSCC, head and neck squamous cell carcinoma; OSCC, Oral squamous cell carcinoma; RT-qPCR, reverse transcription-quantitative PCR.

Lipofectamine $2000^{\circledR}$ reagent (Invitrogen; Thermo Fisher Scientific, Inc.), according to the manufacturer's instructions. Briefly, the cells were seeded into a 12-well plate at 30,000 cells/well and after reaching the logarithmic growth phase, transfection was performed. A plasmid quantity of $1 \mu \mathrm{g}$ was added into $100 \mu \mathrm{l}$ opti-MEM medium (Gibco; Thermo Fisher Scientific, Inc.); $2 \mu 1$ Lipofectamine $2000^{\circledR}$ was then added into $100 \mu$ l opti-MEM medium. Following the plasmid-opti-MEM mixture and Lipofectamine $2000^{\circledR}$-opti-MEM mixture incubation at $25^{\circ} \mathrm{C}$ for $5 \mathrm{~min}$, the two mixtures were added together and incubated at $25^{\circ} \mathrm{C}$ for $20 \mathrm{~min}$. The Lipo2000-plasmid-opti-MEM mixture was then added into the well. After $4 \mathrm{~h}$, the medium was replaced with DMEM containing 10\% FBS. After $24 \mathrm{~h}$, the cells were used in subsequent experimentation.

Luciferase activity. Luciferase activity was measured as previously described (21). Briefly, the CAL 27 cells were seeded into a 12-well plate at 30,000 cells/well. After the cells reached the logarithmic growth phase, $1 \mu \mathrm{g}$ TCTN1 promoter plasmid was transfected into CAL27 cells or SCC15 cells. At $24 \mathrm{~h}$ following transfection, the activity measurement was performed. For activity measurement, the cells were lysed in a cell lysis buffer of the Promega E1500 Luciferase Assay System (Promega Corporation), and all cell lysates were then incubated with luciferin of the Promega E1500 Luciferase Assay System. Luciferase activity was measured with a Berthold SiriusC luminometer (Titertek-Berthold). Renilla luciferase activity was used for normalisation.
Analysis of transcription factors binding to the TCTN1 core promoter. PROMO is a virtual laboratory used for identifying putative transcription factor binding sites, based on DNA sequences (19). The core promoter sequence was submitted to PROMO (http://alggen.lsi.upc.es/cgi-bin/promo_ v3/promo/promoinit.cgi?dirDB=TF_8.3). The dissimilarity level for predicted factors was set as $\leq 5$. The putative transcription factor predicted by PROMO was then submitted to JASPAR2020 software (20) (http://jaspar.genereg.net/), which provided a high-quality transcription factor binding profile database in order to evaluate further the transcription factor binding to the TCTN1 core promoter. The relative profile score threshold was set as $80 \%$, and the transcription factor getting the highest score would be studied further.

Chromatin Immunoprecipitation (ChIP). ChIP assay was performed using a ChIP assay kit (cat. no. P2078, Beyotime Institute of Biotechnology), according to the manufacturer's instructions. Briefly, crosslink was performed by incubating the CAL27 and SCC15 cells in 1\% formaldehyde (Beyotime Institute of Biotechnology) and terminsated by $1 \%$ glycin (Beyotime Institute of Biotechnology). The chromatin was fractured into fragments between 200 and 1,000 bp by ultrasonication at $400 \mathrm{~W}$ for $10 \mathrm{~min}$ in a Ultrasonic Cell Disruptor (JY92-IIN, Ningbo Scientz Biotechnology Co., Ltd.), and was then incubated with the anti-TFAP2A antibody (ab108311; Abcam) (1:1,000 diluted) at $4^{\circ} \mathrm{C}$ for $12 \mathrm{~h}$ and dismantled by Protein A/G MagBeads (Thermo Fisher Scientific, Inc.). The chromatin fragments were then incubated with anti-flag 
A

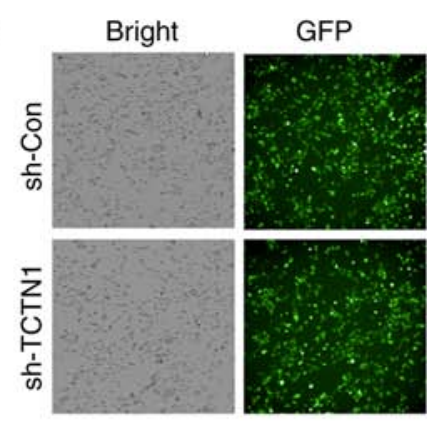

D
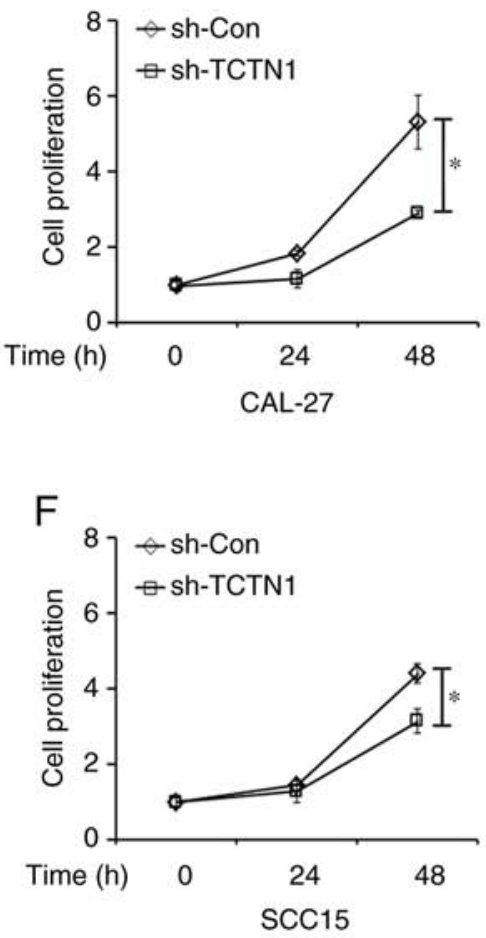

B
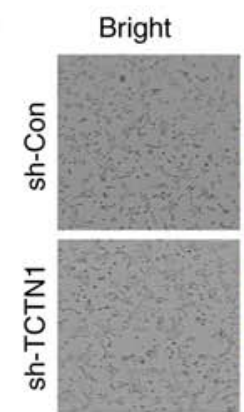

E

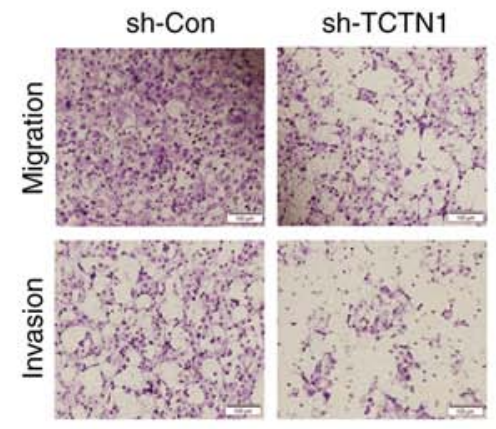

G

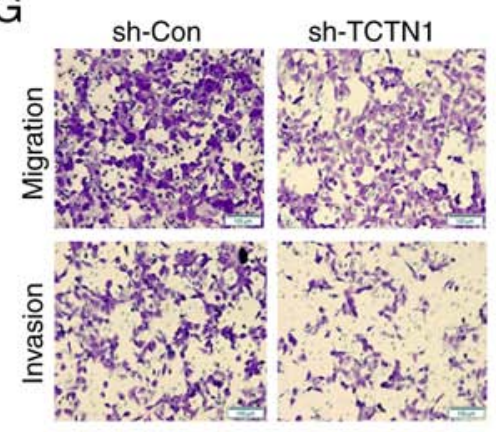

C

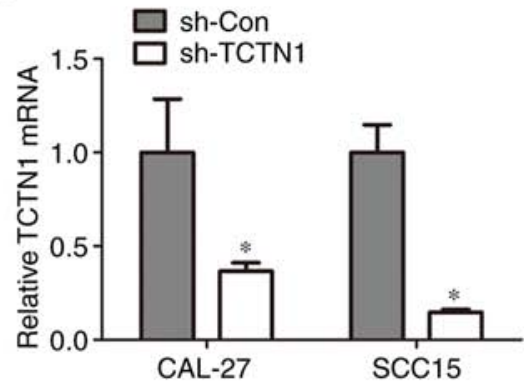

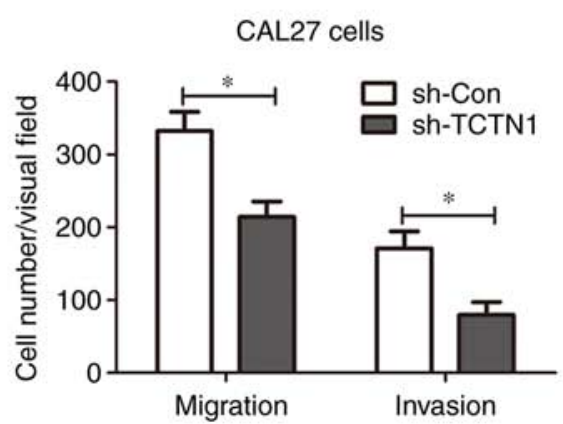

SCC15 cells

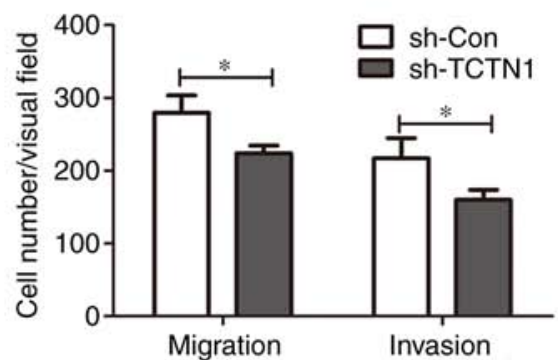

$\mathrm{H}$

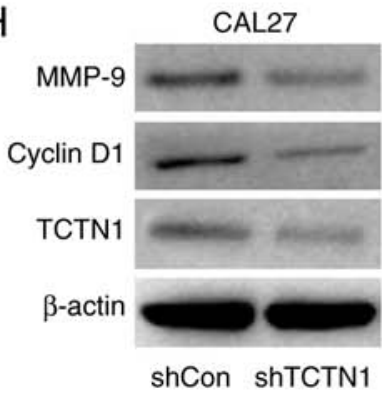

$\operatorname{SCC} 15$

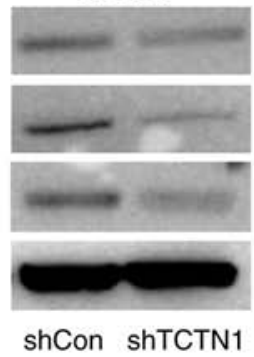

Figure 2. TCTN1 knockdown inhibits OSCC tumor cell proliferation, migration and invasion. (A) CAL-27 cells transfected with lv-sh-Con and lv-shTCTN1 were observed under a fluorescence microscope. (B) SCC15 cells transfected with lv-sh-Con and lv-shTCTN1 were observed under a fluorescence microscope. (C) TCTN1 mRNA expression in CAL-27 cells and SCC15 cells with/without TCTN1 knockdown. ${ }^{*} \mathrm{P}<0.05$ vs. sh-Con. (D) CAL-27 cell proliferation with/without TCTN1 knockdown. "P<0.05 vs. sh-Con. (E) CAL-27 cell migration and invasion capacity with/without TCTN1 knockdown. "P<0.05 vs. sh-Con. (F) SCC15 cell proliferation with/without TCTN1 knockdown, "P<0.05 vs. sh-Con. (G) SCC15 cell migration and invasion capacity with/without TCTN1 knockdown. "P<0.05 vs. sh-Con. (H) CAL27 and SCC15 cell lysates with or without TCTN1 knockdown were extracted and subjected to western blot analysis for TCTN1, MMP-9, and cyclin D1 expression evaluation. GFP, green fluorescent protein; TCTN1, tectonic family member 1; OSCC, Oral squamous cell carcinoma; MMP-9, metalloproteinase 9.

antibody (\#14793; Cell Signaling Technology, Inc.) (1:1,000 diluted) at $4^{\circ} \mathrm{C}$ for $12 \mathrm{~h}$ as a negative control. Following the cross-linking unfastening with the use of $0.2 \mu \mathrm{M} \mathrm{NaCl}$ at $65^{\circ} \mathrm{C}$ for $6 \mathrm{~h}$, the pulled down fragment was subjected to PCR amplification in an ABI Veriti PCR thermocycle instrument (Applied
Biosystems) according to the following protocol: $95^{\circ} \mathrm{C}, 10 \mathrm{~min}$; $\left(95^{\circ} \mathrm{C}, 15 \mathrm{sec} ; 55^{\circ} \mathrm{C}, 15 \mathrm{sec} ; 72^{\circ} \mathrm{C}, 30 \mathrm{sec}\right.$ for 30 cycles; $72^{\circ} \mathrm{C}$, $5 \mathrm{~min}$. The primers for amplifying the fragments containing TFAP2A-binding sites of the TCTN1 promoter were as follows: 5'-CACGCCTGTAATCCCAACTA-3' (forward) and 

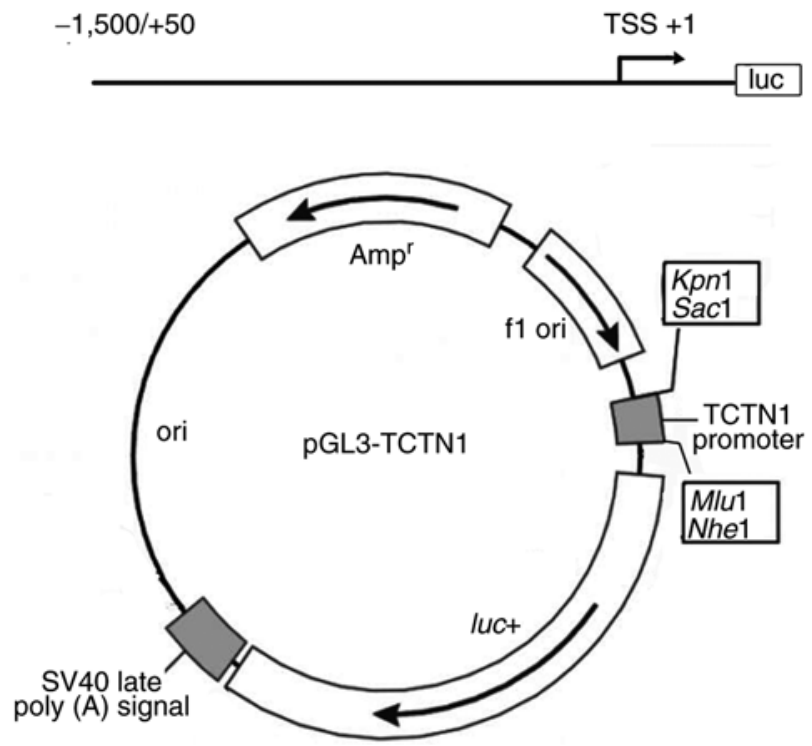

Figure 3. Structure diagram of the TCTN1 promoter-reporter, pGL3-TCTN1. TCTN1, tectonic family member 1 .

5'-GTGAAGCTGGCGTAAACGAG-3' (reverse). The PCR products were analyzed on $1.0 \%$ agarose gel electrophoresis and then photographed on an Ultraviolet luminescence gel imager (Gel Doc XR, Bio-Rad Laboratories, Inc.).

Statistical analysis. The data of CCK-8 assay, transwell assay, RT-qPCR and luciferase assay in the present study were performed in triplicate and the results are presented as the mean $\pm \mathrm{SD}$. Statistical analysis was performed using SPSS 16.0 software (IBM). An unpaired Student's t-test was used to determine the significance of differences between two groups which were not equal in numbers. The significance of the differences in the expression of TCTN1 and TFAP2A between the OSCC tissues and the paired normal tissues was determined using a paired t-test. Tukey's post hoc test following one-way ANOVA was used to evaluate the significance of the differences among multiple independent groups. The correlation between TFAP2A expression and TCTN1 was analyzed using Spearman's rank correlation coefficient analysis with Rho and $\mathrm{P}$-values as indicated. $\mathrm{P}<0.05$ was considered to indicate a statistically significant difference.

\section{Results}

Expression of TCTN1 in HNSCC and OSCC. The association of TCTN1 with the clinicopathological features of patients with HNSCC was analyzed using the UALCAN database (http://ualcan.path.uab.edu/index.html). It was revealed that the TCTN1 expression levels in HNSCC tissues were increased in comparison with those in normal tissue; however, this increase was not statistically significant (Fig. 1A). Furthermore, the expression of TCTN1 in different HNSCC tumor stages was analyzed. The tumor stage was defined by researchers of the UALCAN database according to American Joint Committee on Cancer (AJCC) pathological tumor stage information (24). In total, 443 of the 520 tumor tissues with a defined tumor stage were analyzed. As depicted in Fig. 1B, the
TCTN1 expression levels in TNM stage 2, 3 and 4 tumor tissue groups were higher than those in the stage 1 group. Tumor grade was attributed to the tumor tissues according to tumor differentiation by researchers of the UALCAN database. The TCTN1 expression levels in the grade 2, 3 and 4 groups were higher than those in the grade 1 tumor group. Additionally, TCTN1 expression levels in the grade 3 and 4 tumor groups were significantly higher than those in the grade 1 tumor group (Fig. 1C). These results indicated that TCTN1 was associated with an increased tumor stage and grade of HNSCC.

Considering that OSCC is the main HNSCC subtype, 21 OSCC tissues were obtained and the expression of TCTN1 was also measured in OSCC tumors. As depicted in Fig. 1D, TCTN1 was overexpressed in the OSCC tissues compared with the paired normal tissues. Moreover, the expression of TCTN1 was compared between the HOK cell, a normal oral epithelial cell line, and four OSCC cells lines. In the CAL27, SCC15 and Tca83 OSCC cell lines, higher TCTN1 expression levels were observed in comparison with those in the HOK cells (Fig. 1E). However, in the SCC9 OSCC cell line, the TCTN1 expression levels appread to be similar to those of the HOCK cells.

Knockdown of TCTN1 inhibits the proliferation, migration and invasion of OSCC cells. Since it was observed that TCTN1 was overexpressed in OSCC tissues, it was therefore hypothesized that TCTN1 may participate in tumor OSCC progression. TCTN1 expression was knocked down in the CAL27 and SCC15 OSCC cell lines through Lv-shTCTN1 transfection. The green fluorescence indicated that the CAL-27 cells (Fig. 2A) and SCC15 cells (Fig. 2B) were successfully transfected with the lentiviral vector. TCTN1 expression was then determined using RT-qPCR. It was demonstrated in Fig. 2C that TCTN1 mRNA expression was significantly decreased in the CAL27 cells (62.59\% reduction) and SCC15 cells (85.95\% reduction) transfected with Lv-shTCTN1, as compared with the Lv-shCon-transfected cells. CCK- 8 cell assay was then used to evaluate the cell proliferative capacity. As depicted in Fig. 2D and F, TCTN1 knockdown exerted an inhibitory effect on the proliferation of the CAL-27 and SCC15 cells. Moreover, the cell migratory and invasive capacity was detected using a Transwell assay. Both cell migration and invasion were inhibited by TCTN1 knockdown in the CAL27 and SCC15 cells (Fig. 2E and G). Furthermore, cyclin D1 and matrix metallopeptidase 9 (MMP-9) expression, associated with cell proliferation, migration and invasion was determined. As illustrated in Fig. 2H, the knockdown of TCTN1 decreased the cyclin D1 and MMP-9 protein levels in both the CAL27 and SCC15 cells. These inhibitory effects of TCTN1 on cell proliferation, migration and invasion suggested that TCTN1 may be a tumor-promoting gene and that it may also be a potential target for OSCC therapy; therefore, elaborating the mechanisms through which TCTN1 is regulated would be of utmost importance.

Construction of TCTN1 promoter-reporter. The TCTN1 promoter sequence was obtained from GenBank (https://www. ncbi.nlm.nih.gov/gene/79600). According to the sequence, high-fidelity PCR was performed in order to acquire the TCTN1 promoter fragment $(-1,500 /+50$, transcriptional starting site was marked as +1$)$. A TCTN1 promoter-reporter was constructed by inserting the promoter fragment into the 
A

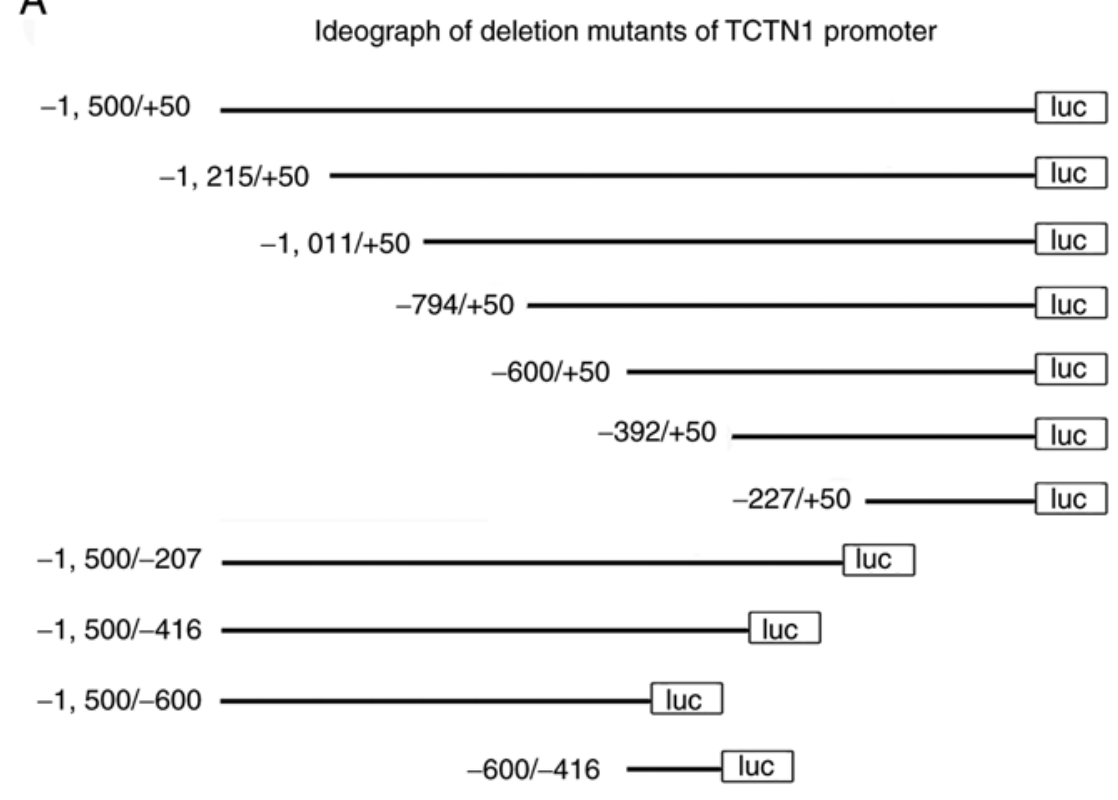

B
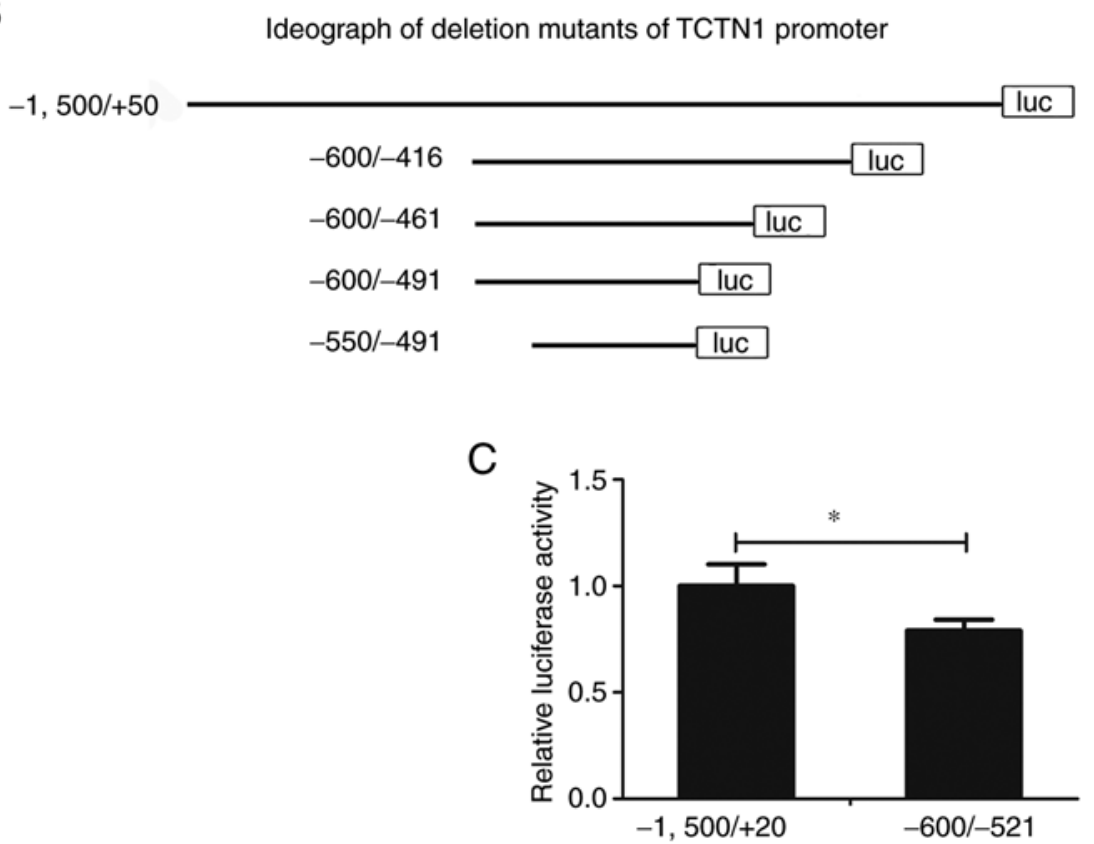

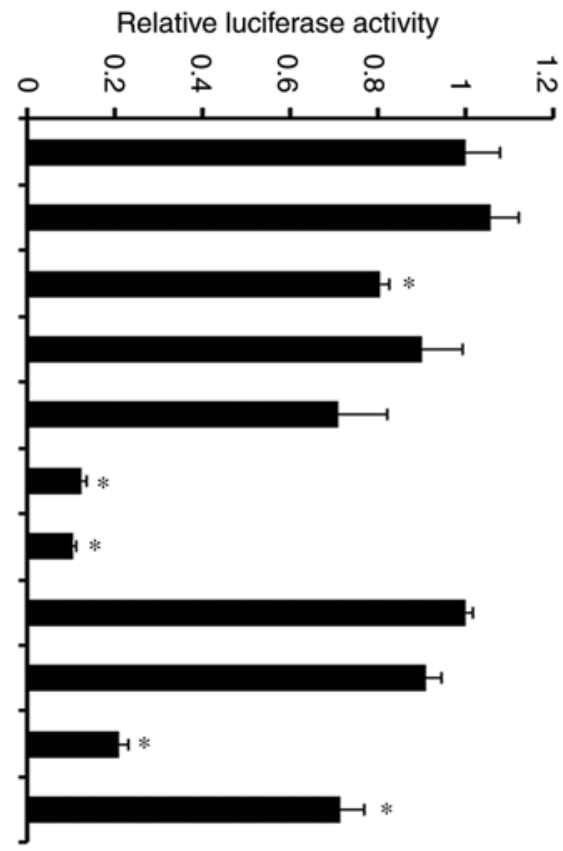

Relative luciferase activity

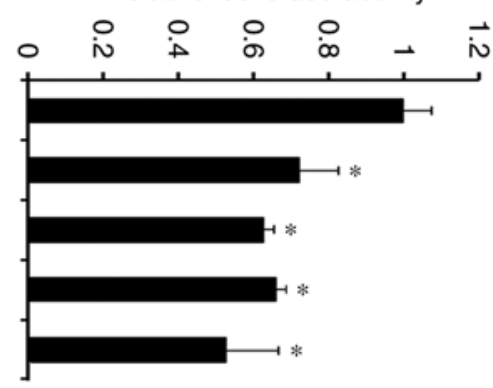

Figure 4. Identification of TCTN1 core promoter. (A) Based on the pGL3-TCTN1, a series of deletion mutants of TCTN1 promoter $(-1,215 /+50,-1,011 /+50$, $-794 /+50,-600 /+50,-392 /+50,-227 /+50,-1,500 /-207,-1500 /-416,-1,500 /-600$ and $-600 /-416)$ were constructed and the luciferase activity was measured. The left panel shows the ideograph of the deletion mutants of TCTN1 promoter; the right panel shows the relative luciferase of each mutant. " $\mathrm{P}<0.05 \mathrm{vs.} \mathrm{the}-1,500 /+50$. (B) Based on the pGL3-TCTN1 (-600/-416), a series of deletion mutants of TCTN1 promoter (-600/-461, -600/-491, and -550/-491) were constructed and the luciferase activity was measured. The left panel depicts the ideograph of the deletion mutants of TCTN1 promoter; the right panel depicts the relative luciferase of each mutant. " $\mathrm{P}<0.05$ vs. the $-1,500 /+50$. (C) Promoter activity of pGL3-TCTN1 $(-1,500 /+50)$ and pGL3-TCTN1 $(-550 /-491)$. "P<0.05. TCTN1, tectonic family member 1 .

pGL3-basic plasmid between SacI and MluI site, and the reporter was named pGL3-TCTN1 (Fig. 3).

Identification of the TCTN1 core promoter. Based on the pGL3-TCTN1, a series of deletion TCTN1 promoter mutants were constructed. These reporters were transfected into the CAL27 cells, and the promoter activity was measured. As demonstrated in Fig. 4A, as the promoter fragment narrowed to
$-600 /-416$, there was still almost $71.32 \%$ activity compared to the full-length promoter $(-1,500 /+50)$, indicating that the core promoter is located in the region of $-600 /-416$. The reporter based on pGL3-CDKL1 (-600/-416) was shortened further and the promoter activity was measured. Even when the promoter narrowed to $-550 /-491$, there was still $63.81 \%$ activity of the full-length promoter, suggesting the region of $-550 /-491$ is the core promoter of TCTN1 (Fig. 4B). This was confirmed further 


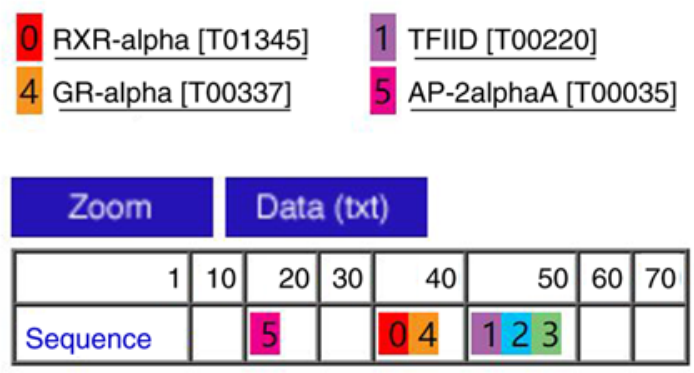

B

\section{Show FASTA Sequence}

$>$ TCTN1 core promoter

TCGCGCTACTGCACTCCAGCCTGGGCGACAGAGTGAGACCCTGTCTCAAAAAAAATCCCC
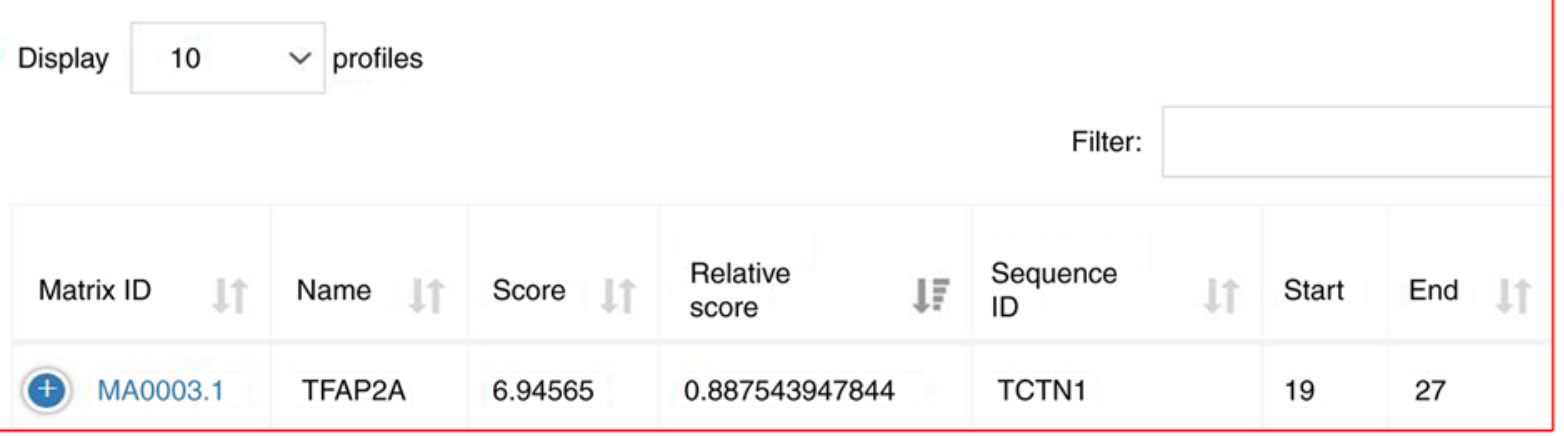

Figure 5. Prediction of putative transcription factors of the TCTN1 core promoter. (A) Six putative transcriptional factors binding to the TCTN1 core promoter were predicted using PROMO software. AP-2alphaA was also termed TFAP2A. (B) TFAP2A can bind to the TCTN1 core promoter, as confirmed using JASPAR2020 software. TCTN1, tectonic family member 1; TFAP2A, transcription factor AP-2 alpha.

in SCC15 cells by comparing the activity of the full-length promoter $(-1,500 /+50)$ and the deletion mutant $(-550 /-491)$ in SCC15 cells. Furthermore, the deletion mutant (-550/-491) demonstrated $\sim 79.06 \%$ the activity of the full-length promoter (Fig. 4C). The aforementioned results indicated that the core promoter of TCTN1 may be located in the -550/-491 region.

Prediction of putative transcription factor of TCTN1 core promoter. The core promoter sequence was uploaded onto PROMO (http://alggen.lsi.upc.es/cgi-bin/promo_ v3/promo/promoinit.cgi?dirDB=TF_8.3). The dissimilarity of predicted factors was set as $\leq 0$. A total of 6 predicted transcriptional factors binding to the TCTN1 core promoter were detected (Fig. 5A). The transcription factors were then submitted to JASPAR 2020, which provides access to a high-quality transcription factor binding profile database. The relative profile score threshold was set as $80 \%$. According to JASPAR2020, only TFAP2A can bind to the TCTN1 core promoter (Fig. 5B). Therefore, it was hypothesized that TFAP2A may be an important transcription factor for TCTN1.

TFAP2A is overexpressed in HNSCC and regulates TCTN1 expression in OSCC cells. The relevance of TFAP2A with the clinicopathological features of patients with HNSCC was then analyzed based on the UALCAN database. The analysis revealed that TFAP2A expression was significantly higher in HNSCC than in normal tissues (Fig. 6A). Furthermore, TFAP2A expression was analyzed in relation to different tumor stages and tumor grades. TFAP2A expression was increased in stage 1,2,3 and 4 tumors compared with normal tissues (Fig. 6B); however no differences in TFPA2 expression levels were observed among stage 1, 2, 3 and 4 tumors. In addition, TFAP2A expression was higher in grade 1, 2, 3 and 4 tumors than in normal tissues; however that between grade 1,2,3 and 4 tumors exhibited no difference (Fig. 6C). TFAP2A expression in the 21 paired OSCC tissues and the corresponding adjacent normal tissues was also confirmed. TFAP2A overexpression was observed in OSCC tissues in comparison with adjacent normal tissues (Fig. 6D). Additionally, the correlation between the TFAP2A and TCTN1 expression levels was also analyzed in the 21 paired tissues and it was demonstrated that TCTN1 expression positively correlated with the TCTN1 expression levels (Fig. 6E). To confirm whether TFAP2A regulates TCTN1 expression, TFAP2A overexpression was induced in the CAL-27 and SCC15 cells, and RT-qPCR was used for TCTN1 expression evaluation. It was observed 

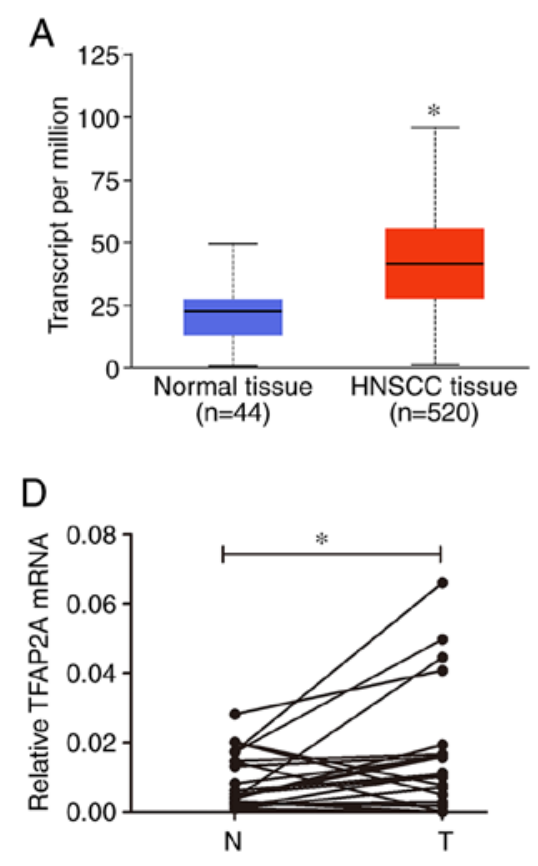

B

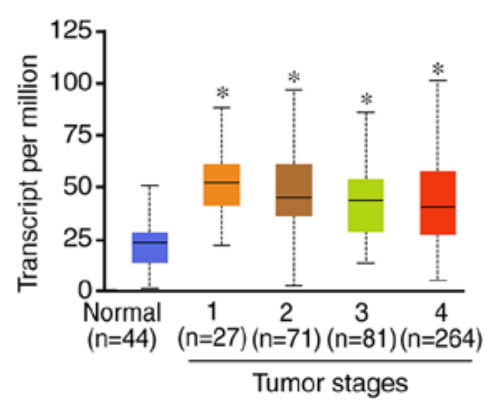

$E$

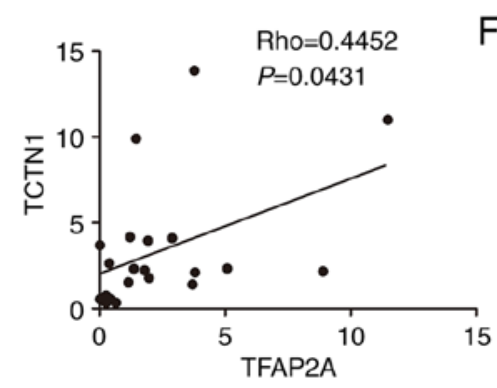

C

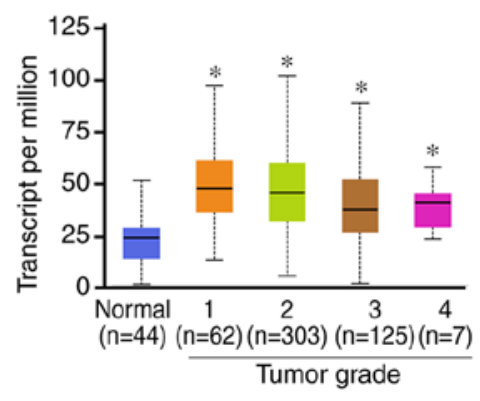

$\mathrm{F}$

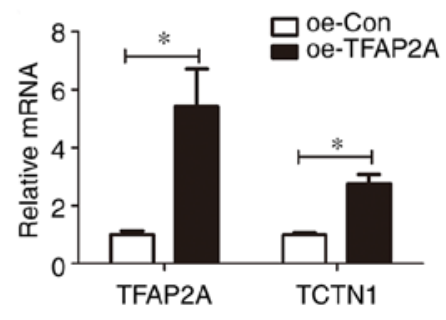

G

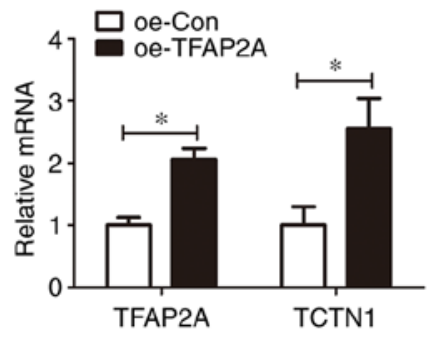

$\mathrm{H}$

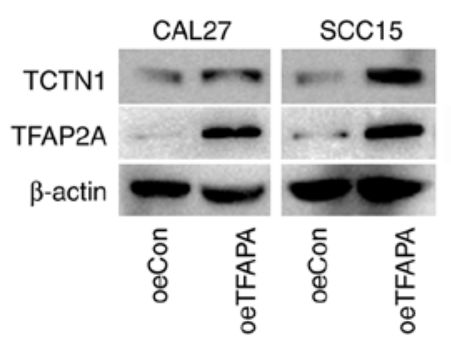

Figure 6. TFAP2A is overexpressed in HNSCC and regulates TCTN1 expression in OSCC cells. (A) TFAP2A expression in normal tissues and HNSCC tissues according to the UALCAN database. (B) TFAP2A expression in different HNSCC tumor stages. "P<0.05 vs. normal. (C) TFAP2A expression in different HNSCC tumor grades. "P $<0.05$ vs. normal. (D) Expression of TFAP2A in 21 OSCC tissues and paired tumor-adjacent normal tissues was detected using RT-qPCR. "P $<0.05$. N, adjacent normal tissue; T, tumor tissue of OSCC. (E) Correlation analysis between TFAP2A expression and TCTN1 expression in 21 paired tissues, with Rho and P-values as indicated. (F) TFAP2A and TCTN1 mRNA expression in CAL-27 cells with/without TFAP2A overexpression; "P<0.05. (G) TFAP2A and TCTN1 mRNA expression in SCC15 cells with/without TFAP2A overexpression; " $\mathrm{P}<0.05$. (H) TFAP2A and TCTN1 protein expression in CAL27 and SCC15 cells with/without TFAP2A overexpression. TFAP2A, transcription factor AP-2 alpha; HNSCC, head and neck squamous cell carcinoma; OSCC, oral squamous cell carcinoma; TCTN1, tectonic family member 1; RT-qPCR, reverse transcription-quantitative PCR.

that TFAP2A was overexpressed 5.38-fold following a Lv-oeTFAP2A infection in CAL-27 cells, and correspondingly induced the upregulation of TCTN1 in the CAL-27 cells (Fig. 6F). Moreover, TFAP2A was overexpressed 2.81-fold with Lv-oeTFAP2A infection, and correspondingly induced the upregulation of TCTN1 in the SCC15 cells. Furthermore, western blot analysis demonstrated that the overexpression of TFAP2A protein levels induced the upregulation of TCTN1 protein levels (Fig. 6H).

TFAP2A regulates TCTN1 transcription in OSCC. The present study then detected whether TFAP2A regulates TCTN1 through the core promoter. TFAP2A was overexpressed 4.912-fold with Lv-oeTFAP2A infection in the CAL-27 cells (Fig. 7A). Subsequently, the core TCTN1 promoter activity in the CAL-27 cells with TFAP2A overexpression was defined. It was observed that TFAP2A overexpression resulted in an increased TCTN1 core promoter activity, indicating that TFAP2A regulated TCTN1 at the transcription level (Fig. 7B). Subsequently, site-directed mutation was performed, in order to mutate the putative binding site of TFAP2A at the TCTN1 core promoter. It was then defined whether the mutations could abolish the effects of TFAP2A overexpression on the TCTN1 promoter activity. The mutant-type of TCTN1 core promoter demonstrated a decreased activity in comparison with the wild-type; additionally, the mutation of the binding site abolished the effect of TFAP2A on the regulation of the TCTN1 core promoter activity (Fig. 7C). In order to confirm that TFAP2A could bind to TCTN1 promoter, ChIP assay was performed. According to the results, there were visible bands in the anti-TFAP2A group, whereas in the anti-flag group, no band was observed, indicating that TFAP2A may bind to the TCTN1 promoter (Fig. 7D).

\section{Discussion}

In the present study, the function of TCTN1 in OSCC cell proliferation, migration and invasion was examined. Moreover, it was demonstrated that the TFAP2A transcription factor may play a main role in the regulation of TCTN1 expression. 

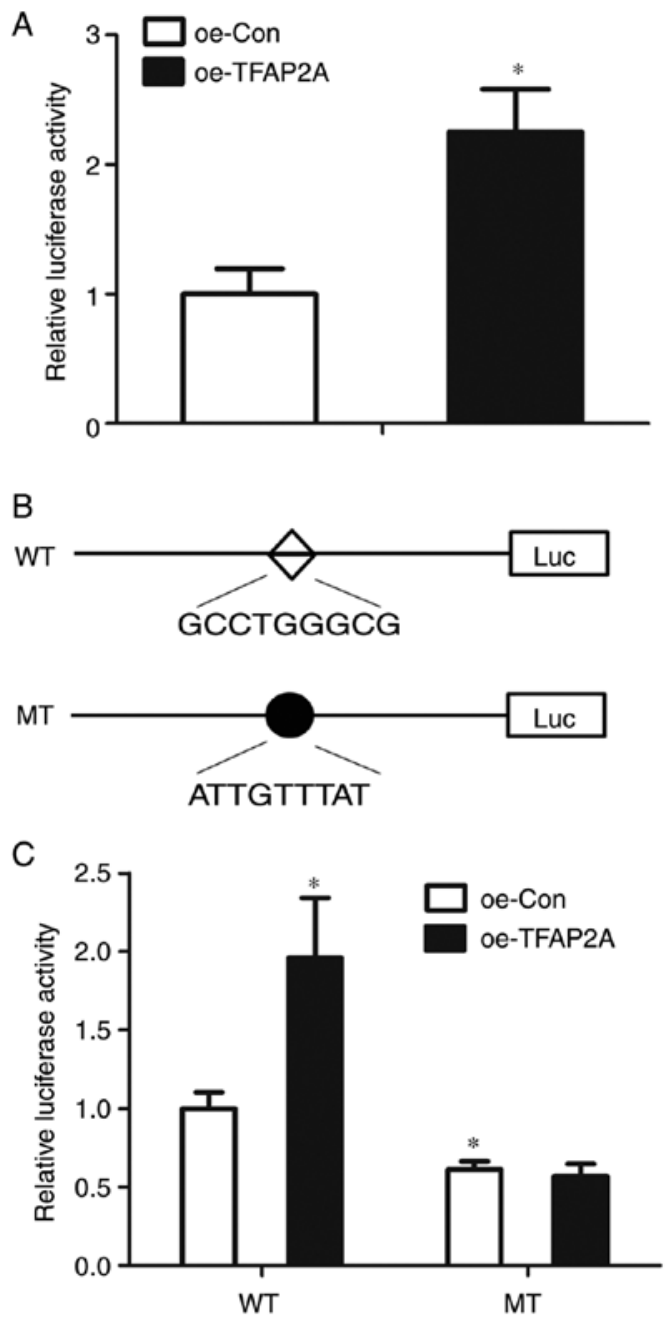

D

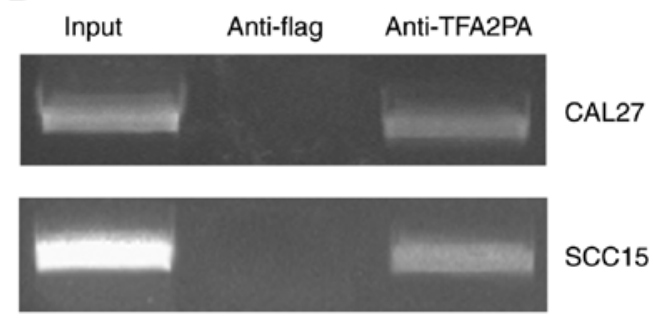

Figure 7. TFAP2A regulates TCTN1 transcription in OSCC. (A) CAL27 cells were transfected with Lv-oeCon or Lv-oeTFAP2A, and the TFAP2A mRNA was detected using RT-qPCR. "P $<0.05$ vs. oe-Con. (B) Luciferase activity of TCTN1 core promoter in CAL-27 cells with/without TFAP2A overexpression. " $\mathrm{P}<0.05$ vs. oe-Con. (C) Luciferase activity of wildtype and mutant type of TCTN1 core promoter in CAL-27 cells with/without TFAP2A overexpression. $\diamond$, TFAP2A binding site in TCTN1 promoter; $\bullet$, mutation of TFAP2A binding site. (D) TFAP2A-TCTN1 promoter interaction evaluation with the use of ChIP assay. Input, PCR amplification using genomic DNA of CAL27 and SCC15 cell as the template. TFAP2A, transcription factor AP-2 alpha; TCTN1, tectonic family member 1; OSCC, Oral squamous cell carcinoma; RT-qPCR, reverse transcription-quantitative PCR; ChIP, chromatin immunoprecipitation assay.

UALCAN is a comprehensive interactive web resource for analyzing cancer OMICS data, by which the relevance of a certain gene with the clinicopathological features of several tumor types can be analyzed (11). Using UALCAN, it was revealed that TCTN1 expression was associated with the tumor stage and grade of HNSCC. Subsequently, it was hypothesized that TCTN1 overexpression in HNSCC in general may also possibly occur in the OSCC subtype. In order to verify this, its expression in OSCC was defined and its function was evaluated. It was observed that TCTN1 was overexpressed in OSCC tumors, as compared with adjacent normal tissues. In addition, it was observed to be overexpressed in three OSCC cells, as compared with the control cells. It was also noted that the SCC9 cell line did not exhibit increased TCTN1 levels, in comparison with the HOK control cell line. This may possibly be attributed to the control cell and the four OSCC cell line origins from different individuals, thus gene expression variations possibly exist among them. Therefore, the effects of alterations of TCTN1 expression on OSCC progression were examined. TCTN1 knockdown resulted in the inhibition of cell proliferation, migration and invasion, indicating that TCTN1 may be a tumor-promoter gene for OSCC progression, suggesting that TCTN1 may be a potential target for OSCC therapy. To the best of our knowledge, this is the first time the involvement of TCTN1 expression in OSCC has been examined. The aforementioned findings, concerning TCTN1 in OSCC progression, are in line with those of previously published studies on other tumor types, including non-small cell lung cancer (25), thyroid cancer (7) and colon cancer cells (8).

Revealing the mechanisms through which cancer-associated genes are regulated is an important approach towards the comprehension of the tumor occurrence-related mechanisms, and the determination of novel therapeutic targets. Several miRNAs have been reported to regulate TCTN1 through the binding at the 3 '-UTR region $(10,26)$. Apart from miRNAs, gene expression is also regulated at the transcriptional level by transcription factors (27). However, the transcriptional regulation of TCTN1 has not been previously reported, at least to the best of our knowledge. The core promoter is the smallest contiguous sequence of DNA in a gene promoter, mediating the initiation of gene transcription (28). In the present study, a TCTN1 promoter-reporter was constructed as well as a serial-deletion mutant promoter-reporter. Considering that the region of $-550 /-491$ exhibited almost $63.8 \%$ of the full-length promoter activity in the CAL27 cells and $79.06 \%$ of the full-length promoter activity in the SCC15 cells, also possessing a binding site of transcription factor IID (TFIID), the core promoter region of TCTN1 was identified, located at $-550 /-491$. This is the first time, to the best of our knowledge, that the core promoter of TCTN1 was located, contributing to the acquisition of key transcriptional factors for TCTN1.

Several putative transcription factors were predicted with the use of PROMO and JASPAR2020 and it was also demonstrated that TFAP2A may be a crucial transcription factor of TCTN1. TFAP2A, also known as AP2A, AP-2alphaA and $\mathrm{AP}-2 \mathrm{TF}$, is an important factor involved in various physiological and pathological processes, including osteogenic differentiation, epidermal development, and cancer progression, such as breast cancer and cervical cancer (29-31). In the present study, it was revealed that TFAP2A upregulated TCTN1 promoter activity and expression in OSCC cells. This may be the first finding of TCTN1 being regulated at the transcription level, to the best of our knowledge.

TFAP2A, a member of the AP-2 family, has been reported to be vital for gene expression regulation. TFAP2A has been reported to activate and inhibit the transcription of target genes (32-34). 
This transcription factor has been mentioned to bind to a specific sequence of the target genes and regulate gene transcription through its interaction with enhancer elements (34). In the present study, it was demonstrated that TFAP2A positively regulated TCTN1 expression, a newly identified target gene of TFAP2A, to the best of our knowledge. TFAP2A plays a crucial role in various crucial biological processes, including melanoma tumor metastasis (35), cell proliferation and development $(36,37)$. The function of TFAP2A in tumor progression has been extensively researched. Different functions of TFAP2A have been reported in carcinogenesis: In prostate cancer, melanoma, hepatocellular cancer, gastric cancer and breast cancer TFAP2A has been associated with a reduced aggressiveness or an improved clinical survival through the regulation of cell growth, differentiation or chemotherapy sensitivity; however, in neuroblastoma, acute myeloid leukemia and several types of squamous cell carcinomas, such as head and neck squamous cell carcinoma, TFAP2A has been reported to promote tumor cell growth and aggressiveness (34).

Subsequently, the putative binding site of TFAP2A in the TCTN1 core promoter was mutated, as predicted using JASPAR2020 software. It was observed that the mutation of the binding site may reduce core promoter activity and completely abolish TFAP2A-regulated TCTN1 core promoter activity, confirming that this site is the binding site of TFAP2A. Blocking this site may abolish the regulatory effects of TFAP2A on TCTN1 and may be a promising OSCC therapeutic target.

A limitation of the present study is that the study was initiated from a pan-cancer gene expression analysis using UALCAN, considering that OSCC is a main type of HNSCC. Subsequently, the association of TCTN1 with OSCC cell proliferation, migration and invasion was analyzed. It was observed that TCTN1 was overexpressed in 21 OSCC tissues in comparison with the paired normal tumor-adjacent tissue. However, the number of tissues enrolled in the present study was insufficient for the analysis of the relevance between TCTN1 and the patient clinicopathological features. Therefore, further clinical analyses are required in future studies, in order to determine the associations between TCTN1 and the patient clinicopathological features. Furthermore, the results were validated in vivo, in order to demonstrate the role of TCTN1 in OSCC progression and to further confirm the transcriptional role of TFAP2A in TCTN1 expression. Additionally, another limitation was that the numbers of patients in the four tumor stage and grade categories differed markedly. The adjustment of the numbers of patients in each of the tumor stage and grade categories and the analysis of the association between TCTN1 expression and tumor stage or grade is required in future studies. However, UALCAN currently provides only gene expression data without any adjustments related to group size. Furthermore, the number of 42 normal tissues in UALCAN is smaller than the number of $520 \mathrm{HNSCC}$ tissues, possibly inducing bias to the results. Therefore, in order to reveal more clearly any relevance between TCTN1 and OSCC, the authors aim to examine a larger cohort including clinicopathological data in future studies, with the inclusion of all necessary group number adjustments.

In total, future studies may involve several steps. Firstly, the relevance between TCTN1 and OSCC may be further elucidated using a multicenter cohort, to confirm whether TCTN1 is an appropriate biomarker for OSCC prognosis. Secondly, downstream factors or signals related to the TCTN1-induced regulation of OSCC proliferation, migration and invasion may be screened using bioinformatics tools and subsequently validated using biomedical laboratory methods. Moreover, TCTN1 was associated with the clinicopathological features of patients with OSCC; however, it was only demonstrated whether TCTN1 regulates OSCC cell proliferation, migration and invasion. In future studies, the possibility of TCTN1 association with other clinicopathological features, including angiogenesis, immune escape, and therapy resistance may be also elucidated.

In conclusion, it was demonstrated in the present study that TCTN1 was overexpressed in OSCC tumors, and was associated with OSCC tumor stage and tumor grade; additionally, the core promoter of TCTN1 was identified and it was determined that TFAP2A may be a crucial transcription factor of TCTN1. The findings of the present study suggested that TFAP2A/TCTN1 may be potential therapeutic targets for OSCC treatment.

\section{Acknowledgements}

Not applicable.

\section{Funding}

The present study was supported by the National Natural Science Foundation of China (grant no. 81602374), the Medical Science and Technology Development Project of Shandong Province (grant no. 2015WS0398), and the Natural Science Foundation of Liaocheng People's Hospital (grant no. LYQN201903).

\section{Availability of data and materials}

Raw data from the experimental results have been uploaded in the Figshare online database (https://figshare. com/s/eda08d428301117387b6). All remaining datasets used and/or analyzed during the present study are available from the corresponding author on reasonable request.

\section{Authors' contributions}

PY designed the study. GB performed the cell culture and western blotting experiments, and drafted the manuscript. NW collected the tissue samples and performed the RT-qPCR experiments, and the construction of TCTN1 promoter-reporter. $\mathrm{CY}$ revised the manuscript and was also involved in the western blotting experiments. FL performed the cell culture experiments. PZ and ZM performed the statistical analysis. BZ performed the Transwell assay. YL performed the ChIP and CCK-8 assays. KX and KL performed the luciferase assay and participated in data analysis. PY and GB confirmed the authenticity of the raw data. All authors have read and approved the final manuscript, and agree to be accountable for all aspects of the present research, ensuring that the accuracy or integrity of any part of the work are appropriately investigated and resolved.

\section{Ethics approval and consent to participate}

OSCC and adjacent normal tissues were collected from pateints at Liaocheng People's Hospital. The tissue collection was checked and approved by the Ethics Committee of 
Liaocheng People's Hospital (approval no. LC202176). All patients provided written informed consent.

\section{Patient consent for publication}

Not applicable.

\section{Competing interests}

The authors declare that they have no competing interests.

\section{References}

1. Almangush A, Makitie AA, Triantafyllou A, de Bree R, Strojan P, Rinaldo A, Hernandez-Prera JC, Suárez C, Kowalski LP, Ferlito A and Leivo I: Staging and grading of oral squamous cell carcinoma: An update. Oral Oncol 107: 104799, 2020.

2. Li J, Wang H,Hang C,Fan Y,Ma Cand Pan Y:Lentivirus-mediated knockdown of TCTN1 inhibits glioma cell proliferation. Appl Biochem Biotechnol 176: 13-21, 2015.

3. Wang C, Li J, Meng Q and Wang B: Three Tctn proteins are functionally conserved in the regulation of neural tube patterning and Gli3 processing but not ciliogenesis and Hedgehog signaling in the mouse. Dev Biol 430: 156-165, 2017.

4. Al-Qattan MM, Shaheen R and Alkuraya FS: Expanding the allelic disorders linked to TCTN1 to include Varadi syndrome (orofaciodigital syndrome type VI). Am J Med Genet A 173: 2439-2441, 2017.

5. Alazami AM, Alshammari MJ, Salih MA, Alzahrani F, Hijazi H, Seidahmed MZ, Abu Safieh L, Aldosary M, Khan AO and Alkuraya FS: Molecular characterization of Joubert syndrome in Saudi Arabia. Hum Mutat 33: 1423-1428, 2012.

6. Gong S, Ji F, Wang B, Zhang Y, Xu X and Sun M: Tectonic proteins are important players in non-motile ciliopathies. Cell Physiol Biochem 50: 398-409, 2018.

7. Xu P, Xia X, Yang Z, Tian Y, Di J and Guo M: Silencing of TCTN1 inhibits proliferation, induces cell cycle arrest and apoptosis in human thyroid cancer. Exp Ther Med 14: 3720-3726, 2017.

8. Dai X, Dong M, Yu H, Xie Y, Yu Y, Cao Y, Kong Z, Zhou B, Xu Y, Yang T and Li K: Knockdown of TCTN1 strongly decreases growth of human colon cancer cells. Med Sci Monit 23: 452-461, 2017.

9. Wang Z, Gao Y, Liu Y, Chen J, Wang J, Gan S, Xu D and Cui X: Tectonic-1 contributes to the growth and migration of prostate cancer cells in vitro. Int J Mol Med 36: 931-938, 2015.

10. Chai L and Yang G: MiR-216a-5p targets TCTN1 to inhibit cell proliferation and induce apoptosis in esophageal squamous cell carcinoma. Cell Mol Biol Lett 24: 46, 2019.

11. Chandrashekar DS, Bashel B, Balasubramanya SAH Creighton CJ, Ponce-Rodriguez I, Chakravarthi BVSK and Varambally S: UALCAN: A portal for facilitating tumor subgroup gene expression and survival analyses. Neoplasia 19: 649-658, 2017.

12. Paul J: Transcriptional regulation in mammalian chromosomes. Symp Soc Exp Biol 25: 117-126, 1971.

13. Ramya Devi KT, Karthik D, Mahendran T, Jaganathan MK and Hemdev SP: Long noncoding RNAs: Role and contribution in pancreatic cancer. Transcription 12: 12-27, 2021.

14. Qiao C, Qiao T, Yang S, Liu L and Zheng $M$ : SNHG17/miR-384/ELF1 axis promotes cell growth by transcriptional regulation of CTNNB1 to activate Wnt/ $\beta$-catenin pathway in oral squamous cell carcinoma. Cancer Gene Ther: Feb 2, 2021 (Epub ahead of print).

15. Jiang H, Chen H, Wan P, Song S and Chen N: Downregulation of enhancer RNA EMX2OS is associated with poor prognosis in kidney renal clear cell carcinoma. Aging (Albany NY) 12: 25865-25877, 2020.

16. Kou XX, Hao T, Meng Z, Zhou YH and Gan YH: Acetylated Sp1 inhibits PTEN expression through binding to PTEN core promoter and recruitment of HDACl and promotes cancer cell migration and invasion. Carcinogenesis 34: 58-67, 2013.

17. Xia CP, Pan T, Zhang N, Guo JR, Yang BW, Zhang D, Li J, $\mathrm{Xu} \mathrm{K}$, Meng Z and He H: Sp1 promotes dental pulp stem cell osteoblastic differentiation through regulating noggin. Mol Cell Probes 50: 101504, 2020.
18. Chan WCW, Tan Z, To MKT and Chan D: Regulation and role of transcription factors in osteogenesis. Int J Mol Sci 22: 5445, 2021.

19. Farre D, Roset R, Huerta M, Adsuara JE, Roselló L, Albà MM and Messeguer X: Identification of patterns in biological sequences at the ALGGEN server: PROMO and MALGEN. Nucleic Acids Res 31: 3651-3653, 2003.

20. Fornes O, Castro-Mondragon JA, Khan A, van der Lee R, Zhang X, Richmond PA, Modi BP, Correard S, Gheorghe M, Baranašić D, et al: JASPAR 2020: Update of the open-access database of transcription factor binding profiles. Nucleic Acids Res 48: D87-D92, 2020.

21. Gan YH and Zhang S: PTEN/AKT pathway involved in histone deacetylases inhibitor induced cell growth inhibition and apoptosis of oral squamous cell carcinoma cells. Oral Oncol 45: e150-e154, 2009.

22. Livak KJ and Schmittgen TD: Analysis of relative gene expression data using real-time quantitative PCR and the 2(-Delta Delta C(T)) method. Methods 25: 402-408, 2001.

23. Xu K, Meng Z, Xian XM, Deng MH, Meng QG, Fang W, Zhang D and Long X: LncRNA PVT1 induces chondrocyte apoptosis through upregulation of TNF- $\alpha$ in synoviocytes by sponging miR-211-3p. Mol Cell Probes 52: 101560, 2020.

24. Amin MB, Greene FL, Edge SB, Compton CC, Gershenwald JE, Brookland RK, Meyer L, Gress DM, Byrd DR and Winchester DP: The eighth edition AJCC cancer staging manual: Continuing to build a bridge from a population-based to a more 'personalized' approach to cancer staging. CA Cancer J Clin 67: 93-99, 2017.

25. Liu W, Wan X, Mu Z, Li F, Wang L, Zhao J and Huang X: MiR-1256 suppresses proliferation and migration of non-small cell lung cancer via regulating TCTN1. Oncol Lett 16: 1708-1714, 2018.

26. Xu X, Gao F, Wang J, Long C, Tao L, Ding L and Ji Y: microRNA-216a-5p inhibits the development of gastric cancer through target combination with TCTN1. Minerva Med: May 29, 2020 (Epub ahead of print).

27. Malik S and Roeder RG: The metazoan mediator co-activator complex as an integrative hub for transcriptional regulation. Nat Rev Genet 11: 761-772, 2010.

28. Chen BS and Hampsey M: Transcription activation: Unveiling the essential nature of TFIID. Curr Biol 12: R620-R622, 2002.

29. Lin X, Yang H, Wang L, Li W, Diao S, Du J, Wang S, Dong R, Li J and Fan Z: AP2a enhanced the osteogenic differentiation of mesenchymal stem cells by inhibiting the formation of YAP/RUNX2 complex and BARX1 transcription. Cell Prolif 52: e12522, 2019.

30. Kousa YA, Fuller E and Schutte BC: IRF6 and AP2A interaction regulates epidermal development. J Invest Dermatol 138: 2578-2588, 2018.

31. Orso F, Fassetta M, Penna E, Solero A, De Filippo K, Sismondi P, De Bortoli M and Taverna D: The AP-2alpha transcription factor regulates tumor cell migration and apoptosis. Adv Exp Med Biol 604: 87-95, 2007.

32. Wang D, Shin TH and Kudlow JE: Transcription factor AP-2 controls transcription of the human transforming growth factor-alpha gene. J Biol Chem 272: 14244-14250, 1997.

33. Liu H, Tan BC, Tseng KH, Chuang CP, Yeh CW, Chen KD, Lee SC and Yung BY: Nucleophosmin acts as a novel AP2alpha-binding transcriptional corepressor during cell differentiation. EMBO Rep 8: 394-400, 2007.

34. Kołat D, Kałuzińska Z, Bednarek AK and Płuciennik E: The biological characteristics of transcription factors AP- $2 \alpha$ and AP- $2 \gamma$ and their importance in various types of cancers. Biosci Rep 39: BSR20181928, 2019.

35. White JR, Thompson DT, Koch KE, Kiriazov BS, Beck AC, van der Heide DM, Grimm BG, Kulak MV and Weigel RJ: AP-2 $\alpha$-mediated activation of E2F and EZH2 drives melanoma metastasis. Cancer Res 81: 4455-4470, 2021.

36. Schorle H, Meier P, Buchert M, Jaenisch R and Mitchell PJ: Transcription factor AP-2 essential for cranial closure and craniofacial development. Nature 381: 235-238, 1996.

37. Nottoli T, Hagopian-Donaldson S, Zhang J, Perkins A and Williams T: AP-2-null cells disrupt morphogenesis of the eye, face, and limbs in chimeric mice. Proc Natl Acad Sci USA 95: 13714-13719, 1998.

This work is licensed under a Creative Commons Attribution-NonCommercial-NoDerivatives 4.0 International (CC BY-NC-ND 4.0) License. 